Pacific

Journal of

Mathematics

EXTENSION OF INCOMPRESSIBLE SURFACES ON THE BOUNDARIES OF 3-MANIFOLDS

Michael Freedman, Hugh Howards, and Ying-Qing Wu 


\title{
EXTENSION OF INCOMPRESSIBLE SURFACES ON THE BOUNDARIES OF 3-MANIFOLDS
}

\author{
Michael Freedman, Hugh Howards, and Ying-Qing Wu
}

\begin{abstract}
An incompressible bounded surface $F$ on the boundary of a compact, connected, orientable 3-manifold $M$ is arcextendible if there is a properly embedded arc $\gamma$ on $\partial M-\operatorname{Int} F$ such that $F \cup N(\gamma)$ is incompressible, where $N(\gamma)$ is a regular neighborhood of $\gamma$ in $\partial M$. Suppose for simplicity that $M$ is irreducible and $F$ has no disk components. If $M$ is a product $F \times I$, or if $\partial M-F$ is a set of annuli, then clearly $F$ is not arc-extendible. The main theorem of this paper shows that these are the only obstructions for $F$ to be arc-extendible.
\end{abstract}

Suppose $F$ is a compact incompressible surface on the boundary of a compact, connected, orientable, irreducible 3-manifold $M$. Let $F^{\prime}$ be a component of $\partial M-\operatorname{Int} F$ with $\partial F^{\prime} \neq \emptyset$. We say that $F$ is arc-extendible (in $F^{\prime}$ ) if there is a properly embedded arc $\gamma$ in $F^{\prime}$ such that $F \cup N(\gamma)$ is incompressible. In this case $\gamma$ is called an extension arc of $F$. We study the problem of which incompressible surfaces on the boundary $M$ are arc-extendible. The result will be used in $[\mathbf{H}]$, in which it is shown that a compact, orientable 3manifold $M$ contains arbitrarily many disjoint, non-parallel, non-boundary parallel, incompressible surfaces, if and only if $M$ has at least one boundary component of genus greater than or equal to two.

Denote by $I$ the unit interval $[0,1]$. We say that $M$ is a product $F \times I$ if there is a homeomorphism $\varphi: M \cong F \times I$ with $\varphi(F)=F \times 1$. Note that in this case $F^{\prime}=\partial M-\operatorname{Int} F$, and $F$ is not arc-extendible. A surface $F$ is diskless if it has no disk component. An incompressible surface with a disk component is always arc-extendible, unless the disk lies on a sphere component of $\partial M$. Thus to avoid trivial cases, we will only consider arcextension of diskless surfaces.

Theorem 1. Let $F$ be a diskless, compact, incompressible surface on the boundary of a compact, connected, orientable, irreducible 3-manifold $M$, and let $F^{\prime}$ be a non-annular component of $\partial M-\operatorname{Int} F$ with $\partial F^{\prime} \neq \emptyset$. Then either $F$ is arc-extendible in $F^{\prime}$, or $M$ is a product $F \times I$.

The proof of the theorem involves some deep results about incompressible surfaces related to Dehn surgery and 2-handle additions. It breaks down into three cases. The case that $F^{\prime}$ is a thrice punctured sphere is treated 
in Theorem 4, which shows that if the surface obtained by gluing $F$ and $F^{\prime}$ along one of the boundary curves of $F^{\prime}$ is compressible for all the three boundary curves of $F^{\prime}$, then $M$ must be a product. The second case is that $F^{\prime}$ is parallel into $F$ (see below for definition). A similar result as above holds in this case. Theorem 9 shows that in the remaining case there is an arc $\gamma$ intersecting some circle $C$ in $F^{\prime}$ at one point, so that all but at most three Dehn twists of $\gamma$ along $C$ are extension arcs of $F$. Moreover, in this case the extension arc $\gamma$ of $F$ can be chosen to have endpoints on any prescribed components of $\partial F^{\prime}$. See Theorem 10 below.

Note that the connectedness and irreducibility of $M$ are irrelevant to the compressibility of surfaces on $\partial M$. However, this does make the conclusion of the theorem simpler. If we drop these assumptions from the theorem, the conclusion should be changed to "Either $F$ is arc-extendible in $F^{\prime}$, or the summand $M^{\prime}$ of the component of $M$ containing $F^{\prime}$ is a product $F^{\prime} \times I$, with $\partial M^{\prime}-\operatorname{Int} F^{\prime}$ a component of $F . "$

Given a simple closed curve $\alpha$ on a surface $S$ on the boundary of $M$, we use $M[\alpha]$ to denote the manifold obtained by adding a 2-handle to $M$ along the curve $\alpha$. More explicitly, $M[\alpha]$ is the union of $M$ and a $D^{2} \times I$, with the annulus $\left(\partial D^{2}\right) \times I$ glued to a regular neighborhood $N(\alpha)$ of $\alpha$ on $\partial M$. Use $S[\alpha]$ to denote the surface on $\partial M[\alpha]$ corresponding to $S$, i.e., $S[\alpha]=(S-N(\alpha)) \cup\left(D^{2} \times \partial I\right)$. The following two lemmas are very useful in dealing with incompressible surfaces. Various versions of Lemma 2 have been proved by Przytycki $[\mathbf{P r}]$, Johannson [Jo], Jaco [Ja], and Scharlemann $[\mathbf{S c h}]$. The lemma as stated is due to Casson and Gordon [CG].

Lemma 2 (The Handle Addition Lemma [CG]). Let $\alpha$ be a simple closed curve on a surface $S$ on the boundary of an orientable irreducible 3-manifold $M$, such that $S$ is compressible and $S-\alpha$ is incompressible. Then $S[\alpha]$ is incompressible in $M[\alpha]$, and $M[\alpha]$ is irreducible.

Lemma 3 (The Generalized Handle Addition Lemma). Let $S$ be a surface on the boundary of an orientable 3-manifold $M$, let $\gamma$ be a 1-manifold on $S$, and let $\alpha$ be a circle on $S$ disjoint from $\gamma$. Suppose $S-\gamma$ is compressible and $S-(\gamma \cup \alpha)$ is incompressible. If $D$ is a compressing disk of $S[\alpha]$ in $M[\alpha]$, then there is a compressing disk $D^{\prime}$ of $S-\alpha$ in $M$ such that $\partial D^{\prime} \cap \gamma \subset \partial D \cap \gamma$.

Proof. This is essentially [Wu2, Theorem 1]. The theorem there stated that $\partial D^{\prime} \cap \gamma$ has no more points than $\partial D \cap \gamma$, but the proof there gives the stronger conclusion that $\partial D^{\prime} \cap \gamma \subset \partial D \cap \gamma$.

We first study the case that the surface $F^{\prime}$ in Theorem 1 is a thrice punctured sphere. Let $\alpha_{1}, \alpha_{2}, \alpha_{3}$ be the boundary curves of $F^{\prime}$. Since $F^{\prime}$ is a component of $\partial M-\operatorname{Int} F$, we have $\alpha_{i} \subset \partial F$ for $i=1,2,3$. Note that 
if $\operatorname{Int} F \cup \operatorname{Int} F^{\prime} \cup \alpha_{i}$ is incompressible for some $i$, then for any essential arc $\gamma$ on $F^{\prime}$ with $\partial \gamma \subset \alpha_{i}$, the surface $F \cup N(\gamma)$ is incompressible. Hence the following theorem proves Theorem 1 in the case that $F^{\prime}$ is a thrice punctured sphere. However, it should be noticed that a similar statement is false if we drop the assumption that $F^{\prime}$ is a sphere with three holes.

Theorem 4. Let $F$ be a diskless compact incompressible surface on the boundary of a compact, connected, orientable, irreducible 3-manifold $M$, and let $F^{\prime}$ be a component of $\partial M-\operatorname{Int} F$ which is a punctured sphere with $\partial F^{\prime}=\alpha_{1} \cup \alpha_{2} \cup \alpha_{3}$. If $\operatorname{Int} F \cup \operatorname{Int} F^{\prime} \cup \alpha_{i}$ is compressible for $i=1,2,3$, then $M$ is a product $F \times I$.

Proof. We fix some notation. Write $\widehat{F}=F \cup F^{\prime}$. Denote by $\widehat{F}_{i}$ the surface obtained by gluing $\operatorname{Int} F$ and $\operatorname{Int} F^{\prime}$ along $\alpha_{i}$, i.e., $\widehat{F}_{i}=\operatorname{Int} F \cup \operatorname{Int} F^{\prime} \cup \alpha_{i}$. Similarly, write $\widehat{F}_{i j}=\operatorname{Int} F \cup \operatorname{Int} F^{\prime} \cup \alpha_{i} \cup \alpha_{j}$.

First notice that $F^{\prime}$ is incompressible. This is because each simple closed curve on $F^{\prime}$ is isotopic to one of the $\alpha_{i} \subset F$, and because $F$ is incompressible and diskless. Since $\operatorname{Int} F \cap \operatorname{Int} F^{\prime}=\emptyset$, the surface $\operatorname{Int} F \cup \operatorname{Int} F^{\prime}$ is incompressible.

Let $M^{\prime}$ be the component of a maximal compression body of $\partial M$ in $M$ which contains $\widehat{F}$. Then a surface on the boundary of $M^{\prime}$ is compressible in $M^{\prime}$ if and only if it is compressible in $M$. Notice that if $M \neq M^{\prime}$, then $M^{\prime}$ is never a product $F \times I$, so if the theorem is true for $M^{\prime}$, it is true for $M$. Hence after replacing $M$ by $M^{\prime}$ if necessary, we may assume without loss of generality that $M$ is a compression body.

We claim that the curves $\alpha_{1}, \alpha_{2}, \alpha_{3}$ are mutually nonparallel on $\widehat{F}$, that is, no component of $F$ is an annulus with both boundary components on $F^{\prime}$. If two curves $\alpha_{1}, \alpha_{2}$, say, are parallel on $\widehat{F}$, then the surface $\operatorname{Int} F \cup \operatorname{Int} F^{\prime}=$ $\widehat{F}-\alpha_{1} \cup \alpha_{2} \cup \alpha_{3}$ is incompressible if and only if $\widehat{F}_{1}=\widehat{F}-\alpha_{2} \cup \alpha_{3}$ is incompressible. However, by assumption $\widehat{F}_{1}$ is compressible, and we have shown that $\operatorname{Int} F \cup \operatorname{Int} F^{\prime}$ is incompressible. Hence the claim follows.

Since $\widehat{F}_{i}$ is compressible, and $\widehat{F}_{i}-\alpha_{i}=\operatorname{Int} F \cup \operatorname{Int} F^{\prime}$ is incompressible, we can apply the Handle Addition Lemma (Lemma 2) to $\widehat{F}_{i}$ and $\alpha_{i}$ to conclude that after adding a 2-handle along $\alpha_{i}$, the surface $\widehat{F}_{i}\left[\alpha_{i}\right]$ is incompressible in $M\left[\alpha_{i}\right]$, and $M\left[\alpha_{i}\right]$ is irreducible.

Consider the surface $\widehat{F}\left[\alpha_{1}\right]$. Notice that after adding the 2-handle, the surface $F^{\prime}$ becomes an annulus on $\widehat{F}\left[\alpha_{1}\right]$ with boundary $\alpha_{2} \cup \alpha_{3}$, so the two curves $\alpha_{2}, \alpha_{3}$ are parallel on $\widehat{F}\left[\alpha_{1}\right]$. Thus, $\widehat{F}_{1}\left[\alpha_{1}\right]=\widehat{F}\left[\alpha_{1}\right]-\alpha_{2} \cup \alpha_{3}$ being incompressible in $M\left[\alpha_{1}\right]$ implies that $\widehat{F}\left[\alpha_{1}\right]-\alpha_{2}$ is incompressible in $M\left[\alpha_{1}\right]$. With the above notation, this says that $\widehat{F}_{13}\left[\alpha_{1}\right]$ is incompressible in $M\left[\alpha_{1}\right]$. 
By assumption $\widehat{F}_{3}$ is compressible in $M$. Let $D$ be a compressing disk of $\widehat{F}_{3}$ in $M$. Then $\partial D$ is disjoint from $\alpha_{1} \cup \alpha_{2}$, because $\partial D \subset \widehat{F}_{3}$. Also, $\partial D$ is not isotopic to $\alpha_{1}$ in $\widehat{F}_{13}$, otherwise $\alpha_{1}$ would bound a disk in $M$, contradicting the assumption that $F$ is diskless and incompressible. We have shown that $\widehat{F}_{13}\left[\alpha_{1}\right]$ is incompressible in $M\left[\alpha_{1}\right]$, so $D$ is not a compressing disk of $\widehat{F}_{13}\left[\alpha_{1}\right]$ in $M\left[\alpha_{1}\right]$, and hence $\partial D$ must bound a disk in $\widehat{F}_{13}\left[\alpha_{1}\right]$. This is true if and only if $\partial D$ is coplanar to $\alpha_{1}$ on $\widehat{F}_{13}$, that is, either $\partial D$ is parallel to $\alpha_{1}$, or it bounds a once punctured torus $T$ in $\widehat{F}_{13}$ which contains $\alpha_{1}$ as a nonseparating curve. The first possibility has been ruled out, so the second must be true. Let $\widehat{T}$ be the torus $T \cup D$. Since we have assumed above that $M$ is a compression body, either (i) $\widehat{T}$ is parallel to a boundary component of $M$, or (ii) $\widehat{T}$ bounds a solid torus, or (iii) $\widehat{T}$ lies in a 3 -ball and bounds a cube with a knotted hole. But since $T$ lies on $\partial M$, a pair of generators of $H_{1}(T)$ cannot both be null homologous in $M$, hence (iii) cannot happen.

If $\widehat{T}$ is parallel to a boundary component $T_{0}$ of $M$, then after adding the 2-handle, the surface $\widehat{T}\left[\alpha_{1}\right]$ becomes a sphere which separates $T_{0}$ from $\widehat{F}\left[\alpha_{1}\right]$, hence is a reducing sphere of $M\left[\alpha_{1}\right]$, which contradicts the irreducibility of $M\left[\alpha_{1}\right]$. Similarly, if $\widehat{T}$ bounds a solid torus $V$ but $\alpha_{1}$ is not a longitude of $V$, then after adding the 2-handle the manifold would have a lens space or $S^{2} \times S^{1}$ summand, which again contradicts the irreducibility of $M\left[\alpha_{1}\right]$. (Note that $M\left[\alpha_{1}\right]$ cannot be a lens space because it has some boundary components.)

We have now shown that there is a compressing disk $D$ of $\widehat{F}_{3}$ in $M$ which cuts the manifold into two pieces, one of which is a solid torus $V$ which contains $\alpha_{1}$ as a longitude, but is disjoint from $\alpha_{2}$. Let $D_{1}$ be a meridian disk of $V$. Then $\partial D_{1} \cap \alpha_{1}$ is a single point, and $\partial D_{1}$ is disjoint from $\alpha_{2}$ because $\partial V$ is disjoint from $\alpha_{2}$. Notice that $\partial D_{1}$ is not coplanar to $\alpha_{2}$, for if $\partial D_{1}$ were parallel to $\alpha_{2}$ then $\alpha_{2}$ would also intersect $\alpha_{1}$, and if $\partial D_{1}$ were to bound a once punctured torus containing $\alpha_{2}$ then $\partial D_{1}$ would be a separating curve on $\partial M$, so it would intersect $\alpha_{1}$ in an even number of points, either case leading to a contradiction. Thus, after adding a 2-handle to $M$ along $\alpha_{2}$, the disk $D_{1}$ remains a compressing disk of $\widehat{F}\left[\alpha_{2}\right]$. Since the two curves $\alpha_{1}$ and $\alpha_{3}$ are parallel in $\widehat{F}\left[\alpha_{2}\right]$, and since $D_{1}$ intersects $\alpha_{1}$ in a single point, we can isotope $D_{1}$ to another disk $D_{2}$ in $M\left[\alpha_{2}\right]$ so that it intersects each of $\alpha_{1}$ and $\alpha_{3}$ in a single point. We are looking for such a disk in $M$; however $D_{2}$ is not necessarily the one because it may intersect the attached 2-handle.

Recall that the surface $\widehat{F}_{2}$ is compressible, but the surface $\widehat{F}_{2}-\alpha_{2}=$ $\operatorname{Int} F \cup \operatorname{Int} F^{\prime}$ is incompressible. Hence we can apply the Generalized Handle Addition Lemma (Lemma 3, with $S=\widehat{F}, \gamma=\alpha_{1} \cup \alpha_{3}$, and $\alpha=\alpha_{2}$ ) to conclude that there is also a compressing disk $D_{3}$ of $\widehat{F}$ in $M$, such that $\partial D_{3}$ is disjoint from $\alpha_{2}$, and $\partial D_{3} \cap\left(\alpha_{1} \cup \alpha_{3}\right)$ is a subset of $\partial D_{2} \cap\left(\alpha_{1} \cup \alpha_{3}\right)$. 
The set $\partial D_{3} \cap\left(\alpha_{1} \cup \alpha_{3}\right)$ is nonempty, otherwise, since $\partial D_{3}$ is also disjoint from $\alpha_{2}, D_{3}$ would be a compressing disk of $\operatorname{Int} F \cup \operatorname{Int} F^{\prime}$, contradicting the incompressibility of $\operatorname{Int} F \cup \operatorname{Int} F^{\prime}$. Since $\alpha_{1} \cup \alpha_{2} \cup \alpha_{3}$ is separating on $\widehat{F}$, the curve $\partial D_{3}$ can not intersect $\alpha_{1} \cup \alpha_{2} \cup \alpha_{3}$ at a single point. It follows that $\partial D_{3} \cap\left(\alpha_{1} \cup \alpha_{3}\right)=\partial D_{2} \cap\left(\alpha_{1} \cup \alpha_{3}\right)$, that is, $\partial D_{3}$ intersects each of $\alpha_{1}, \alpha_{3}$ in a single point. Such a disk is called a bigon.

Denote by $D_{13}$ the bigon $D_{3}$ above. Interchanging the roles of $\alpha_{1}$ and $\alpha_{2}$ in the above argument, we get another compressing disk $D_{23}$ of $\widehat{F}$ in $M$, which is disjoint from $\alpha_{1}$, and intersects each of $\alpha_{2}, \alpha_{3}$ in a single point. Using the fact that no compressing disk of $\widehat{F}$ would intersect $\alpha_{1} \cup \alpha_{2} \cup \alpha_{3}$ at a single point, we can modify $D_{13}$ and $D_{23}$ by a simple innermost circle outermost arc disk swapping argument, so that $D_{13}$ and $D_{23}$ are disjoint, and still have the same number of intersection points with each $\alpha_{i}$. Cutting $M$ along $D_{13} \cup D_{23}$, we get a submanifold $M^{\prime}$ of $M$, in which the surface $F^{\prime}$ becomes a disk $\widetilde{F}^{\prime} \subset F^{\prime}$, and the surface $F$ becomes a surface $\widetilde{F} \subset F$. It is clear that one boundary component $C$ of $\widetilde{F}$ bounds a disk on $\partial M^{\prime}$, namely the union of $\widetilde{F}^{\prime}$ and the two copies of $D_{13} \cup D_{23}$. Since $F$ is incompressible, this curve $C$ bounds a disk in $F$, so $\widetilde{F}$ must be a disk. These disks together form a sphere boundary component of $M^{\prime}$. Since $M$ is connected and irreducible, $M^{\prime}$ must be a 3 -ball, so it is a product $\widetilde{F} \times I$. Gluing back along $D_{13}$ and $D_{23}$, we see that $M$ is a product $F \times I$. This completes the proof of Theorem 4 .

Below, $F, F^{\prime}$ and $M$ will be as in Theorem 1 . Using Theorem 4 we may assume that $F^{\prime}$ is not a thrice punctured sphere. A curve $C^{\prime}$ on $F^{\prime}$ is $\partial$ nonseparating if (i) $C^{\prime}$ is not parallel to a boundary curve on $F^{\prime}$, and (ii) there is a proper arc $\gamma$ in $F^{\prime}$ intersecting $C^{\prime}$ in a single point. A sub-surface $G^{\prime}$ of $F^{\prime}$ is parallel into $F$ if there is a product $G^{\prime} \times I \subset M$ such that $G^{\prime}=G^{\prime} \times 0$, and $G^{\prime} \times 1 \subset F$. Similarly, a curve $C^{\prime}$ on $F^{\prime}$ is parallel into $F$ if there is an embedded annulus $A \subset M$ with $\partial A=C^{\prime} \cup C$, where $C \subset F$.

Lemma 5. If $F^{\prime}$ is compressible, then there is a d-nonseparating curve $C^{\prime}$ on $F^{\prime}$ which is not parallel into $F$.

Proof. Let $D$ be a compressing disk of $F^{\prime}$. If $\partial D$ is nonseparating on $F^{\prime}$, let $C^{\prime}$ be a curve in $F^{\prime}$ that intersects $\partial D$ in one point. Then $C^{\prime}$ is nonseparating, hence $\partial$-nonseparating on $F^{\prime}$. We want to show that $C^{\prime}$ is not parallel into $F$. Otherwise, let $A$ be an annulus with $\partial A=C^{\prime} \cup C$, where $C \subset F$. Then $A \cap D$ is a proper 1-manifold on $D$. But $\partial(A \cap D)=(\partial A) \cap \partial D$ is a single point, which is absurd. Hence $C^{\prime}$ is the curve required. 
Now assume that $\partial D$ is separating on $F^{\prime}$, cutting $F^{\prime}$ into $F_{1}^{\prime}$ and $F_{2}^{\prime}$. Choose a simple loop $C_{i}$ on $F_{i}^{\prime}$ as follows. If $F_{i}^{\prime}$ is nonplanar, then it contains a pair of nonseparating curves intersecting each other in one point, at least one of which is not null-homologous in $M$. Choose this one as $C_{i}$. If $F_{i}^{\prime}$ is planar, choose $C_{i}$ to be isotopic to a boundary curve of $F^{\prime}$. Note that since $F$ is incompressible and diskless, $C_{i}$ is not null-homotopic in $M$. Also notice that in all cases there is a properly embedded $\operatorname{arc} \gamma$ on one of the $F_{i}^{\prime}$ which intersects $C_{1} \cup C_{2}$ in one point.

Now choose a band $B=I \times I$ on $F^{\prime}$ such that $B \cap \partial D=I \times \frac{1}{2}, B \cap C_{1}=$ $I \times 0, B \cap C_{2}=I \times 1$, and $B$ is disjoint from the arc $\gamma$ above. Such a band exists because $\gamma$ is a nonseparating arc on $F_{i}^{\prime}$. Let $C^{\prime}$ be the band sum of $C_{1}$ and $C_{2}$, that is, $C^{\prime}=\left(C_{1} \cup C_{2}-I \times\{0,1\}\right) \cup(\{0,1\} \times I)$. Then $C^{\prime}$ intersects $\gamma$ in one point. Since $C^{\prime}$ intersects $\partial D$ essentially in two points, it is not parallel to any boundary component on $F^{\prime}$. Therefore $C^{\prime}$ is $\partial$-nonseparating.

We want to show that $C^{\prime}$ is not parallel into $F$. Using the property that $C_{i}$ are not null-homotopic in $M$, one can show by an innermost circle argument that $C^{\prime}$ is not null-homotopic in $M$. Now suppose that there is an annulus $A$ in $M$ with $\partial A=C^{\prime} \cup C$, where $C \subset F$. Since $C^{\prime}$ is not null-homotopic in $M, A$ is incompressible in $M$. By surgery along an innermost circle of $D \cap A$ one can eliminate all circle intersections of $A \cap D$. Since $\partial(A \cap D)$ consists of two points, $A \cap D$ is a single arc, which has endpoints on the same component of $\partial A$, hence it cuts off a disk $D^{\prime}$ from $A$. Assume without loss of generality that $D^{\prime} \cap F^{\prime}$ is on $F_{1}^{\prime}$. Let $D^{\prime \prime}$ be the disk on $D$ bounded by $(A \cap D) \cup(B \cap D)$, and let $B_{1}=B \cap F_{1}^{\prime}$. Then $D^{\prime} \cup D^{\prime \prime} \cup B_{1}$ is a disk with boundary $C_{1}$, which contradicts the fact that $C_{1}$ is not null-homotopic in $M$. Therefore, $C^{\prime}$ is not parallel into $F$.

Lemma 6. Suppose $F^{\prime}$ is incompressible, and is not a thrice punctured sphere. Then either (i) there is a d-nonseparating curve $C^{\prime}$ on $F^{\prime}$ which is not parallel into $F$, or (ii) $F^{\prime}$ is parallel into $F$.

Proof. Since $F^{\prime}$ is not a thrice punctured sphere, one can easily find a $\partial$-nonseparating curve $\alpha_{0}$ on $F^{\prime}$. Assume that (i) is not true, so all $\partial$ nonseparating curves are parallel into $F$. We want to show that $F^{\prime}$ is parallel into $F$.

Since $\alpha_{0}$ is parallel into $F$, the annulus $N\left(\alpha_{0}\right)$ is also parallel into $F$. It is an incompressible annulus because $\alpha_{0}$ is essential on $F^{\prime}$ and $F^{\prime}$ is incompressible. Among all connected incompressible surfaces in $\operatorname{Int} F^{\prime}$ which contain $\alpha_{0}$ and are parallel into $F$, choose $G^{\prime}$ such that the complexity $\left(\chi\left(G^{\prime}\right),\left|\partial G^{\prime}\right|\right)$ is minimal in the lexicographic order, where $\chi\left(G^{\prime}\right)$ is the Euler characteristic of $G^{\prime}$, and $\left|\partial G^{\prime}\right|$ is the number of boundary components of $G^{\prime}$. All incompressible sub-surfaces of $F^{\prime}$ have Euler characteristics bounded below by $\chi\left(F^{\prime}\right)$, hence such $G^{\prime}$ does exist. 
If all boundary components of $G^{\prime}$ are parallel to some boundary components on $F^{\prime}$, then either $G^{\prime}$ is contained in a collar of $\partial F^{\prime}$, or $F^{\prime}-$ Int $G^{\prime}=\partial F^{\prime} \times I$. The first case does not happen because $G^{\prime}$ contains the $\partial$ nonseparating curve $\alpha_{0}$, which by definition is not parallel to any boundary curve on $F^{\prime}$. In the second case $F^{\prime}$ is isotopic to $G^{\prime}$, so it is parallel into $F$, and we are done. Hence we may assume that some boundary curve $\beta$ of $G^{\prime}$ is not parallel to any boundary curve on $F^{\prime}$.

We want to find a $\partial$-nonseparating curve $\alpha^{\prime}$ which intersects $\beta$ essentially in one or two points. If $\beta$ is nonseparating on $F^{\prime}$, choose $\alpha^{\prime}$ to be any curve on $F^{\prime}$ that intersects $\beta$ in a single point. Then $\alpha^{\prime}$ is nonseparating, hence $\partial$-nonseparating on $F^{\prime}$. If $\beta$ separates $F^{\prime}$ into $F_{1}^{\prime}$ and $F_{2}^{\prime}$, choose an essential $\operatorname{arc} \alpha_{i}^{\prime}$ on $F_{i}^{\prime}$ with $\partial \alpha_{1}^{\prime}=\partial \alpha_{2}^{\prime} \subset \beta$. Moreover, if $F_{i}^{\prime}$ is nonplanar, choose $\alpha_{i}^{\prime}$ to be nonseparating on $F_{i}^{\prime}$. Then $\alpha^{\prime}=\alpha_{1}^{\prime} \cup \alpha_{2}^{\prime}$ is $\partial$-nonseparating, and intersects $\beta$ essentially in two points, as required.

Isotope $\alpha^{\prime}$ so that it intersects $\partial G^{\prime}$ minimally. The geometric intersection number between $\alpha^{\prime}$ and $\beta$ is 1 or 2 , so $\alpha^{\prime} \cap \partial G^{\prime} \neq \emptyset$. Since $\alpha^{\prime}$ is $\partial$-nonseparating, by our assumption above it is parallel into $F$, so there is an annulus $A$ with $\partial A=\alpha^{\prime} \cup \alpha$, where $\alpha \subset F$. Isotope $A$ rel $\alpha^{\prime}$ so that it intersects $\left(\partial G^{\prime}\right) \times I$ minimally. Since $G^{\prime}$ is incompressible, $\left(\partial G^{\prime}\right) \times I$ consists of incompressible annuli in $M$, hence $A \cap\left(\left(\partial G^{\prime}\right) \times I\right)$ has no trivial circles. Since $F$ and $F^{\prime}$ are also incompressible, one can show that $A \cap\left(\left(\partial G^{\prime}\right) \times I\right)$ has no trivial arcs on $A$ either. Therefore $A \cap\left(\left(\partial G^{\prime}\right) \times I\right)$ consists of vertical $\operatorname{arcs}\left(\alpha^{\prime} \cap \partial G^{\prime}\right) \times I$. These arcs cut $A$ into several squares $\alpha_{i}^{\prime} \times I$, where each $\alpha_{i}^{\prime}$ is the closure of a component of $\alpha^{\prime}-\partial G^{\prime}$. Choose $i$ so that $\alpha_{i}^{\prime}$ lies outside of $G^{\prime}$. Let $H$ be the component of $F^{\prime}-\operatorname{Int} G^{\prime}$ that contains $\alpha_{i}^{\prime}$. Then $G^{\prime \prime}=G^{\prime} \cup N\left(\alpha_{i}^{\prime}\right)$ is a surface parallel into $F$, and $\chi\left(G^{\prime \prime}\right)=\chi\left(G^{\prime}\right)-1$. The arc $\alpha_{i}^{\prime}$ is essential on $H$, so the only case that some boundary component $\gamma$ of $G^{\prime \prime}$ bounds a disk on $F^{\prime}$ is when $H$ is an annulus, and $\gamma$ is the boundary of the disk obtained by cutting $H$ along $\alpha_{i}^{\prime}$. Since $F$ and $F^{\prime}$ are incompressible and $M$ is irreducible, both ends of the annulus $\gamma \times I \subset G^{\prime \prime} \times I \subset M$ bound disks on $F \cup F^{\prime}$, which together with $\gamma \times I$ bounds a 3-ball in $M$. It follows that $G^{\prime} \cup H$ is parallel into $F$. Since $G^{\prime} \cup H$ has the same Euler characteristic as $G^{\prime}$ but fewer boundary components, this contradicts the choice of $G^{\prime}$. Therefore $\partial G^{\prime \prime}$ consists of essential curves on $F^{\prime}$. Since $F^{\prime}$ is incompressible, $G^{\prime \prime}$ is also incompressible. Since $\chi\left(G^{\prime \prime}\right)<\chi\left(G^{\prime}\right)$, this again contradicts the choice of $G^{\prime}$.

Given a simple closed curve $\alpha$ and a proper arc $\gamma$ on $F^{\prime}$, denote by $\tau_{\alpha}^{n} \gamma$ the curve obtained from $\gamma$ by Dehn twisting $n$ times along $\alpha$, and by $N\left(\tau_{\alpha}^{n} \gamma\right)$ a regular neighborhood of $\tau_{\alpha}^{n} \gamma$ on $\partial M$. Suppose $T$ is a fixed torus boundary component of a 3-manifold $M$. Denote by $M(r)$ the manifold obtained by Dehn filling on $T$ along a slope $r$ on $T$, that is $M(r)$ is obtained by gluing a solid torus $V$ to $M$ along $T$ so that the curve $r$ on $T$ bounds a meridian 
disk in $V$. Denote by $\Delta\left(r_{1}, r_{2}\right)$ the minimal geometric intersection number between two slopes $r_{1}, r_{2}$. The following two theorems will be used in the proof of Theorem 9, which proves Theorem 1 in the case that $F^{\prime}$ contains a $\partial$-nonseparating curve which is not parallel into $F$.

Lemma 7 ([Wu2, Theorem 1]). Let $T$ be a torus component on the boundary of a 3-manifold $M$, and let $S$ be an incompressible surface on $\partial M-T$. Suppose there is no incompressible annulus in $M$ with one boundary component on each of $S$ and $T$. If $S$ is compressible in $M\left(r_{1}\right)$ and $M\left(r_{2}\right)$, then $\Delta\left(r_{1}, r_{2}\right) \leq 1$. In particular, $S$ is incompressible in all but at most three $M(r)$.

The following lemma can be found in $[\mathbf{M}]$, $[\mathbf{S h}]$ or $[\mathbf{C G L S}$, Theorem 2.4.3].

Lemma 8. Let T, S, $M$ be as in Lemma 7, and assume further that $M$ is irreducible. Suppose that there is an incompressible annulus $A$ in $M$ with one boundary component on $S$ and the other a curve $r_{0}$ on $T$. Then either $S$ is a torus and $M=S \times I$, or $S$ remains incompressible in all $M(r)$ with $\Delta\left(r, r_{0}\right)>1$.

Theorem 9. Let $\alpha$ be a $\partial$-nonseparating curve on $F^{\prime}$ which is not parallel into $F$, and let $\gamma$ be a proper arc on $F^{\prime}$ intersecting $\alpha$ in one point. Then $F_{n}=F \cup N\left(\tau_{\alpha}^{n} \gamma\right)$ is incompressible for all but at most three consecutive $n$ 's.

Proof. Let $K$ be the knot obtained by pushing $\alpha$ slightly into $M$. There is an embedded annulus $A_{0}$ in $M$ with $\partial A_{0}=\alpha \cup K$. Consider the manifold $M_{K}=M-\operatorname{Int} N(K)$, where $N(K)$ is a regular neighborhood of $K$ in $M$. Let $T$ be the torus $\partial N(K)$, and let $(m, l)$ be the meridian-longitude pair on $T$ such that $l=A_{0} \cap T$. Denote by $M_{K}(p / q)$ the manifold obtained by Dehn filling on $T$ along the slope $p m+q l$. The Dehn twist $\tau_{\alpha}^{-n}$ on $F^{\prime}$ extends to a Dehn twist of $M_{K}$ along the annulus $A=A_{0} \cap M_{K}$, which sends the meridian slope $m$ of $T$ to the slope $m-n l$, so it extends to a homeomorphism $\varphi_{n}: M=M_{K}(1 / 0) \cong M_{K}(-1 / n)$, which maps the curve $\tau_{\alpha}^{n} \gamma$ to the curve $\gamma$, and hence the surface $F_{n}$ to the surface $F_{0}=F \cup N(\gamma)$. It follows that $\varphi_{n}$ is a homeomorphism of pairs

$$
\varphi_{n}:\left(M, F_{n}\right) \rightarrow\left(M_{K}(-1 / n), F_{0}\right) .
$$

Therefore to prove the theorem we need only show that for all but at most three consecutive integers $n$, the surface $F_{0}$ is incompressible in $M_{K}(-1 / n)$.

Claim 1. $T=\partial N(K)$ is incompressible in $M_{K}$, and $M_{K}$ is irreducible.

If $D$ is a compressing disk of $T$ in $M_{K}$, then $\partial D$ must intersect the meridian $m$ of $K$ in one point, because otherwise after the trivial Dehn filling, $M=M_{K}(1 / 0)$ would contain a lens space or $S^{2} \times S^{1}$ summand, contradicting the irreducibility of $M$. It follows that $K$, and hence $\alpha$, bounds a disk 
in $M$. In this case $\alpha$ is parallel to a trivial curve on $F$, which contradicts the assumption that $\alpha$ is not parallel into $F$. Similarly, if $M_{K}$ is reducible, then since $M$ is irreducible, $K$ is contained in a ball in $M$, so $\alpha$ would be null-homotopic. Using Dehn's Lemma, we see that $\alpha$ bounds a disk in $M$, hence is parallel to a trivial circle in $F$, contradicting the assumption that $\alpha$ is not parallel into $F$.

Claim 2. $F_{0}$ is incompressible in $M_{K}$.

Recall that $A$ denotes the annulus $A_{0} \cap M_{K}$. Since $\alpha$ intersects $\gamma$ in a single point, $A \cap F_{0}$ is a single arc $C$ on the boundary curve $\alpha$ of $A$. Let $D$ be a compressing disk of $F_{0}$, chosen so that $|D \cap A|$, the number of components in $D \cap A$, is minimal. After disk swapping along disks on $A$ bounded by innermost circles, we can assume that no component of $D \cap A$ is a trivial circle on $A$. Since $T$ is incompressible by Claim 1, the annulus $A$ is also incompressible, so $D \cap A$ contains no essential circle component on $A$ either. Hence $D \cap A$ consists of arcs only. If some arc $e$ of $D \cap A$ is parallel in $A$ to a sub-arc on $C=A \cap F_{0}$, then after boundary compressing $D$ along a disk $\Delta$ cut off by an outermost such arc we will get two disks $D_{1}, D_{2}$ with boundary on $F_{0}$, at least one of which has boundary an essential curve on $F_{0}$, hence is a compressing disk of $F_{0}$. Since $\left|D_{i} \cap A\right|<|D \cap A|$, this contradicts the minimality of $|D \cap A|$. Therefore, each arc of $D \cap A$ is essential relative to $C$, in the sense that it is not parallel in $A$ to an arc on $C$. See Figure 1(a). Notice that $|D \cap A| \neq 0$, otherwise $D$ would be a compressing disk of $F$, contradicting the incompressibility of $F$.

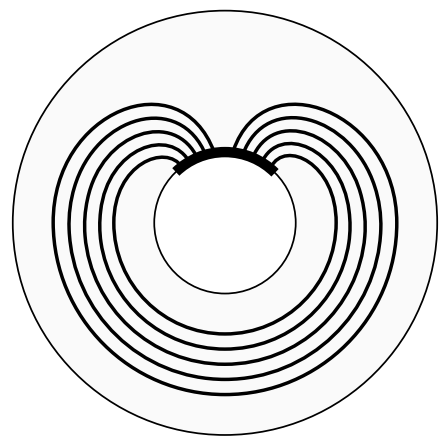

(a)

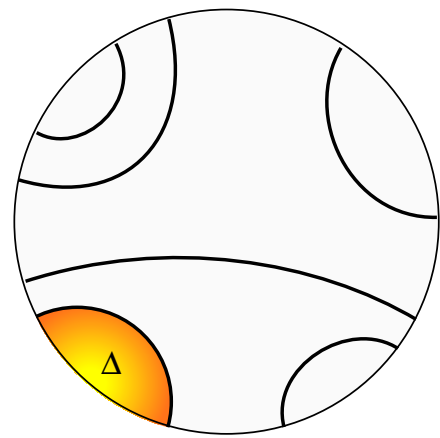

(b)

Figure 1.

Consider an outermost disk $\Delta$ on $D$, as shown in Figure $1(\mathrm{~b})$. Then $\partial \Delta$ consists of two $\operatorname{arcs} e_{1}, e_{2}$, where $e_{1}$ is an arc on $A$ which is essential relative 
to $C$, and $e_{2}$ is an arc on $F_{0}$ with interior disjoint from $C$. Thus $e_{2} \cap N(\gamma)$ consists of two $\operatorname{arcs} e_{2}^{\prime}, e_{2}^{\prime \prime}$. Let $t_{1}$ be the subarc of $C$ connecting the two ends of $e_{2}^{\prime} \cup e_{2}^{\prime \prime}$ on $C$, and let $t_{2}$ be the subarc on $\partial N(\gamma)$ connecting the other two ends of $e_{2}^{\prime} \cup e_{2}^{\prime \prime}$. Then $e_{2}^{\prime} \cup t_{1} \cup e_{2}^{\prime \prime} \cup t_{2}$ bounds a disk $\Delta^{\prime}$ on $N(\gamma)$. Now $A^{\prime}=\Delta \cup \Delta^{\prime}$ is an annulus in $M$, with one boundary component $e_{1} \cup t_{1}$ an essential circle on $A$, which is parallel to $\alpha$, and the other component $\widehat{e}_{2} \cup t_{2}$ a curve on $F$, where $\widehat{e}_{2}$ is the closure of $e_{2}-\left(e_{2}^{\prime} \cup e_{2}^{\prime \prime}\right)$. This contradicts the assumption that $\alpha$ is not parallel into $F$.

Claim 3. There is no incompressible annulus $P$ in $M_{K}$ with one boundary component $C_{1}$ on $F_{0}$ and the other component $C_{2}$ a curve on $T$ which is disjoint from $l=A \cap T$.

The proof is similar to that of Claim 2. Choose $P$ so that $|P \cap A|$ is minimal. Using the fact that $P$ is incompressible, one can show as above that $P \cap A$ has no trivial circle component. Note that since $C_{2}$ is disjoint from $l, P \cap A$ has no arc component with endpoints on $l=A \cap T$. If $P \cap A$ had some essential circle component, choose such a component $t$ which is closest to $l$ on $A$. By cutting and pasting along the annulus on $A$ bounded by $t \cup l$, one would get another incompressible annulus $P^{\prime}$ which has fewer intersection components with $A$. As in the proof of Claim 2 one can eliminate all arc components of $P \cap A$ which on $A$ are inessential relative to $C=A \cap F_{0}$. Hence $P \cap A$ consists of arcs with ends on $C$ and are essential relative to $C$, as shown in Figure 1(a). Also, since $P$ is disjoint from $l, P \cap A$ consists of inessential arcs on $P$. Now one can use a disk $\Delta$ cut off by an outermost arc on $P$, proceed as in the proof of Claim 2 to get an annulus with one boundary on $\alpha$ and the other on $F$, and get a contradiction. Finally, if $P \cap A=\emptyset$ then $P$ extends to an annulus with one boundary on $\alpha$ and the other on $F$, contradicting the assumption that $\alpha$ is not parallel into $F$. This completes the proof of Claim 3.

We now continue with the proof of Theorem 9 . We have shown that $F_{0}$ is incompressible in $M_{K}$. If there is no essential annulus in $M_{K}$ with one boundary component on each of $F_{0}$ and $T$, then by Lemma 7 we know that $F_{0}$ is incompressible in $M_{K}(r)$ for all but at most three slopes $r$ with mutual intersection number 1. In particular, it is incompressible in $M_{K}(-1 / n)$ for all but at most two consecutive $n$ 's, so the theorem follows. Now suppose there is an essential annulus $P$ in $M_{K}$ with one boundary component on $F_{0}$ and the other a curve $r_{0}$ on $T$. Since $F_{0}$ is not a closed surface, it is not a torus. Hence by Lemma $8, F_{0}$ remains incompressible in $M_{K}(-1 / n)$ unless $\Delta\left(-1 / n, r_{0}\right) \leq 1$. By Claim $3, r_{0}$ is not the longitude slope $0 / 1$, therefore, $\Delta\left(-1 / n, r_{0}\right) \leq 1$ holds for at most three consecutive integers $n$. This completes the proof of Theorem 9 . 
Proof of Theorem 1. By Theorem 4, Lemmas 5 and 6, and Theorem 9, we can now assume that $F^{\prime}$ is incompressible and is parallel into $F$. We want to show that either $F$ is arc-extendible in $F^{\prime}$, or $M$ is a product $F \times I$. As in the proof of Theorem 4, we may assume without loss of generality that $M$ is a compression body, so all closed incompressible surfaces of $M$ are boundary parallel. Let $\alpha_{1}, \ldots, \alpha_{k}$ be the boundary curves of $F^{\prime}$. Let $F^{\prime} \times I$ be a product in $M$ such that $F^{\prime}=F^{\prime} \times 0$ and $F^{\prime} \times 1 \subset F$. Write $\alpha_{i}^{1}=\alpha_{i} \times 1$, which is a curve on $F$ isotopic to $\alpha_{i}$ in $M$.

We have assumed above that $F^{\prime}$ is incompressible in $M$, so $\operatorname{Int} F \cup \operatorname{Int} F^{\prime}$ is incompressible in $M$. Write $\widehat{F}_{i}=\operatorname{Int} F \cup \operatorname{Int} F^{\prime} \cup \alpha_{i}$. If $\widehat{F}_{i}$ is incompressible for some $i$, then $F \cup N(\gamma)$ is incompressible for any essential arc $\gamma$ in $F^{\prime}$ with endpoints on $\alpha_{i}$, and we are done. (Such an arc exists because $F^{\prime}$ is not an annulus or disk.) So assume that $\widehat{F}_{i}$ is compressible for all $i$. By the Handle Addition Lemma (Lemma 2), after adding a 2-handle to $M$ along $\alpha_{i}$, the surface $\widehat{F}_{i}\left[\alpha_{i}\right]$ is incompressible, and $M\left[\alpha_{i}\right]$ is irreducible. Notice that since $F^{\prime}$ is incompressible, the curve $\alpha_{i}^{1}=\alpha_{i} \times 1$ in $F$ is essential on $F$. But after adding the 2-handle, $\alpha_{i}^{1}$ bounds a disk in $M\left[\alpha_{i}\right]$, so it must also bound a disk on $\widehat{F}_{i}\left[\alpha_{i}\right]$ because $\widehat{F}_{i}\left[\alpha_{i}\right]$ is incompressible. By definition $\widehat{F}_{i}\left[\alpha_{i}\right]$ is obtained from ( $\left.\operatorname{Int} F \cup \operatorname{Int} F^{\prime}\right)-\operatorname{Int} N\left(\alpha_{i}\right)$ by capping off the two copies of $\alpha_{i}$ with disks, hence $\alpha_{i}^{1} \cup \alpha_{i}$ bounds an annulus $A_{i}$ on $\widehat{F}_{i}$. Denote by $A_{i}^{\prime}$ the annulus $\alpha_{i} \times I \subset F^{\prime} \times I \subset M$. Then $T_{i}=A_{i} \cup A_{i}^{\prime}$ is a torus in $M\left(T_{i}\right.$ cannot be a Klein bottle since $M$ is a compression body). Since we have assumed above that $M$ is a compression body, either (i) $T_{i}$ bounds a solid torus $V_{i}$, or (ii) it is parallel to some torus component of $\partial M$, or (iii) it lies in a ball and bounds a cube with knotted hole. Since $F^{\prime}$ is incompressible, the curve $\alpha_{i}$ is not null homotopic in $M$, hence (iii) does not happen. Since $M\left[\alpha_{i}\right]$ is irreducible, one can proceed as in the proof of Theorem 4 to show that $T_{i}$ must bound a solid torus $V_{i}$ with $\alpha_{i}$ a longitude. This is true for all $i$. It is now easy to see that $M$ is a product $F \times I$.

The following theorem supplements Theorem 1. It says that in most cases there are extension arcs with endpoints on any prescribed boundary compponents of $F^{\prime}$.

Theorem 10. Let $F, F^{\prime}, M$ be as in Theorem 1. Suppose $M$ is not a product $F \times I$, and suppose $F^{\prime}$ is not parallel into $F$ and is not a thrice punctured sphere. Then it contains an extension arc $\gamma$ of $F$ with endpoints on any prescribed components of $\partial F^{\prime}$.

Proof. We need to find a curve $\alpha$ on $F^{\prime}$ which is not parallel into $F$. If $F^{\prime}$ is nonplanar, then by the proof of Lemmas 5 and 6, we can find such an $\alpha$ (denoted by $C^{\prime}$ there) on $F^{\prime}$ which is actually nonseparating on $F^{\prime}$. Hence given any boundary components $\partial_{1}, \partial_{2}$ of $F^{\prime}$, (possibly $\partial_{1}=\partial_{2}$ ), there is an arc $\gamma$ with endpoints on $\partial_{1}$ and $\partial_{2}$, intersecting $\alpha$ in one point. By Theorem 
9 , for all but at most three integers $n$, the $\operatorname{arc} \gamma_{n}=\tau_{\alpha}^{n} \gamma$ is an extension arc of $F$.

Now suppose $F^{\prime}$ is planar with $\left|\partial F^{\prime}\right| \geq 4$. First assume that $\partial_{1}, \partial_{2}$ are distinct boundary components of $F^{\prime}$. By the proofs of Lemmas 5 and 6 , there is a curve $\alpha$ which is a band sum of two boundary components of $F^{\prime}$, such that $\alpha$ is not parallel into $F$. From the proofs one can see that we can always choose $\alpha$ to be the band sum of $\partial_{1}$ and $\partial_{3}$, with $\partial_{3} \neq \partial_{1}, \partial_{2}$. Hence there is an arc $\gamma$ from $\partial_{1}$ to $\partial_{2}$ intersecting $\alpha$ in one point. We can then apply Theorem 9 to get an extension $\operatorname{arc} \gamma_{n}$ with one endpoint on each of $\partial_{1}$ and $\partial_{2}$.

We now proceed to find an extension arc in $F^{\prime}$ with boundary on the same component $\partial_{1}$ of $\partial F^{\prime}$. By the proof of Lemmas 5 and 6 , we can choose the curve $\alpha$ above to be the band sum of of $\partial_{2}$ and $\partial_{3}$, with $\partial_{1} \neq \partial_{2}, \partial_{3}$. Recall that $\alpha$ is not parallel into $F$. Choose an arc $\gamma$ as follows. Let $\partial_{2}^{\prime}$ be a curve on $F^{\prime}$ parallel to $\partial_{2}$, let $\gamma^{\prime}$ be an arc connecting $\partial_{2}^{\prime}$ to $\partial_{1}$ intersecting $\alpha$ in one point, and let $Q$ be the sub-surface $N\left(\gamma^{\prime} \cup \partial_{2}^{\prime}\right)$ of $F^{\prime}$. Then $\gamma$ is the closure of the arc component of $\partial Q \cap \operatorname{Int} F^{\prime}$, that is, $\gamma$ is the arc component of the frontier of $Q$ in $F^{\prime}$, see Figure 2 below. Consider the surface $F_{0}=F \cup N(\gamma)$, and observe that $F_{0}$ is isotopic to the surface $F \cup Q$. After Dehn twisting along $\alpha$, it is isotopic to the surface $F \cup N\left(\tau_{\alpha}^{n} \gamma\right)$; hence to show that all but at most three $\tau_{\alpha}^{n} \gamma$ are extension arcs of $F$ in $F^{\prime}$, we need only show that $F \cup Q$ is incompressible after all but at most three Dehn twists along $\alpha$. Since $F \cup Q$ intersects $\alpha$ in a single arc, the argument in the proof of Theorem 9 is still valid, with the following easy modifications. We use the notations in that proof.

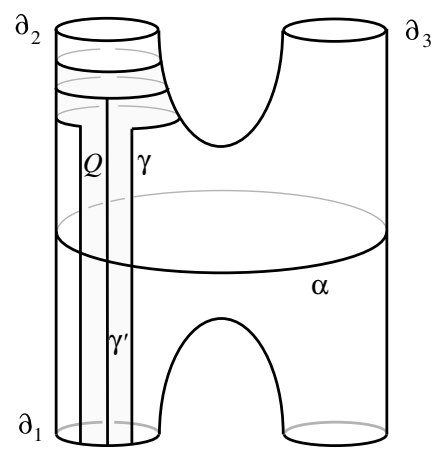

Figure 2.

The proof of Claim 2 needs the following modifications. (i) The arc $e_{2}$ on the boundary of the outermost disk $\Delta$ may be on $Q$. In this case, notice 
that the other $\operatorname{arc} e_{1}$ on $\partial \Delta$ is isotopic to an arc $\alpha_{1}$ on $\alpha$, and $e_{2} \cup \alpha_{1}$ is isotopic in $F^{\prime}$ to the curve $\partial_{3}$, so the fact that $e_{1} \cup e_{2}$ bounds a disk $\Delta$ would imply that $\partial_{3}$ bounds a disk. Since $\partial_{3}$ is also on $\partial F$, this contradicts the fact that $F$ is incompressible and diskless. (ii) The compressing disk $D$ of $F \cup Q$ could be disjoint from the annulus $A$. But since $F$ is incompressible, this would imply that $\partial D$ lies on $Q$, hence is isotopic to $\partial_{2}$, which would imply that $\partial_{2}$ bounds a disk, again contradicting the assumption that $F$ is incompressible and diskless.

The proof of Claim 3 applies to show that the annulus $P$ there can be modified to be disjoint from the annulus $A$. Then notice that the component of $\partial P$ on $F \cup Q$ is either in $F$, or in $Q$ and hence parallel to $\partial_{2}$. Since $\partial_{2} \subset F$, in either case $P$ can be extended to an annulus with one boundary component on $\alpha$ and the other on $F$, which contradicts the assumption that $\alpha$ is not parallel into $F$.

The rest of the proof of Theorem 9 follows verbatim to show that $F \cup Q$ is incompressible after all but at most three Dehn twists along $\alpha$.

Remark. Theorem 10 is not true if $F^{\prime}$ is a thrice punctured sphere.

\section{References}

[CG] A. Casson and C. Gordon, Reducing Heegaard splittings, Topology Appl., 27 (1987), 275-283.

[CGLS] M. Culler, C. Gordon, J. Luecke and P. Shalen, Dehn surgery on knots, Annals Math., 125 (1987), 237-300.

[H] H. Howards, Generating Disjoint Incompressible Surfaces, Preprint, 1998.

[Ja] W. Jaco, Adding a 2-handle to 3-manifolds: An application to property $R$, Proc. Amer. Math. Soc., 92 (1984), 288-292.

[Jo] K. Johannson, On surfaces in one-relator 3-manifolds, Low-Dimensional Topology and Kleinian Groups, London Math. Soc. Lecture Notes Ser., 112 (1984), 157-192.

[M] W. Menasco, Closed incompressible surfaces in alternating knot and link complements, Topology, 23(1) (1984), 37-44.

[Pr] J. Przytycki, Incompressibility of surfaces after Dehn surgery, Michigan Math. J., 30 (1983), 289-308.

[Sch] M. Scharlemann, Outermost forks and a theorem of Jaco, Combinatorial methods in topology and algebraic geometry (Rochester, N.Y., 1982), Contemp. Math., 44, 189-193.

[Sh] M. Short, Some closed incompressible surfaces in knot complements which survive surgery, Low Dimensional Topology, London Math. Soc. Lecture Note Ser., 95 (1985), 179-194.

[Wu1] Y-Q. Wu, A generalization of the handle addition theorem, Proc. Amer. Math. Soc., 114 (1992), 237-242. 
[Wu2] Incompressibility of surfaces in surgered 3-manifolds, Topology, 31 (1992), 271-279.

Received June 16, 1998 and revised February 1, 1999. The first author was partially supported by an NSF grant. The third author's research at MSRI was supported in part by NSF grant \#DMS 9022140.

Microsoft Research

1 Microsoft WAY

Redmond, WA 98053

E-mail address: michaelf@microsoft.com

WAKe Forest University

Winston-SAlEm, NC 27109

E-mail address: howards@wfu.edu

UNIVERSITY OF IOWA

IowA City, IA 52242

E-mail address: wu@math.uiowa.edu 